\title{
Conception fonctionnelle de services d'entreprise fondée sur l'alignement entre cour de métier et Système d'Information
}

\author{
Jacques Simonin* — Philippe Picouet* — Jean-Marc Jézéquel** \\ * Telecom Bretagne / Institut Télécom - CS 83818 - F-29238 Brest - France \\ Lab STICC UMR CNRS 3254 \\ Université Européenne de Bretagne \\ \{jacques.simonin,philippe.picouet\}@telecom-bretagne.eu \\ ** IRISA et Université de Rennes 1 \\ Campus universitaire de Beaulieu \\ F-35042 Rennes \\ jezequel@irisa.fr
}

RÉSUMÉ. La connaissance des processus constituant le cœur de métier de l'entreprise et sa structure organisationnelle permet d'aligner l'organisation de cette entreprise sur sa stratégie. Or pour concevoir un ensemble de services fournis par l'entreprise à un client particulier, il faut être capable de décrire les processus métier du point de vue client, et non de la production. Afin de respecter les objectifs de l'entreprise, nous proposons dans cet article de fonder la démarche de conception des services, informatisés ou non, sur le point de vue des systèmes de l'architecture d'entreprise ou EA (Enterprise Architecture). L'apport de l'EA à notre démarche de conception des services d'entreprise est conditionné par l'écart entre la prise en compte de la stratégie de l'entreprise au niveau du SI cible et sa prise en compte au niveau du cœur de métier. L'alignement ainsi défini est complété dans cette démarche de conception par une conception automatique des services grâce à l'ingénierie des modèles.

ABSTRACT. The enterprise organization must fulfil its strategy. Processes describing enterprise business core and enterprise organizational structure enable enterprise to meet this objective. This paper concerns business process driven design of customer oriented services. The description of business processes from customer instead of the production department allows indeed service set providing to the customer by the enterprise. In order to satisfy enterprise objectives, the service design procedure is so based on the Enterprise Architecture (EA) system point of view, whether services are computerized or not. Our service design procedure benefits from EA according to the enterprise strategy realization gap between the target Information System and the business core. This service design procedure adds an enterprise service automated design thanks to model driven engineering to the alignment so defined.

MOTS-CLÉS : processus métier, service d'entreprise, architecture d'entreprise, alignement de modèles, ingénierie des modèles.

Revue. Volume $\mathrm{X}-\mathrm{n}^{\circ} \mathrm{x} /$ année, pages 1 à $\mathrm{X}$ 
KEYWORDS: business process, enterprise service, Enterprise Architecture, model alignment, model driven engineering.

\section{Introduction}

En plus de l'organisation qui la caractérise, l'entreprise est identifiée depuis une quinzaine d'année par les processus qui décrivent son cœur de métier et non plus à partir de la décomposition, interne à l'entreprise, du travail en tâches telle que créée par Adam Smith en 1776. Cette identification de l'entreprise est conforme à l'approche de réingénierie où le processus est défini comme une série d'activités qui, ensemble, produisent une valeur pour un client (Hammer et al., 1993).

D'autre part, face aux évolutions de la stratégie d'une entreprise, l'urbanisation de son système d'information (SI), cadre de l'EA (Enterprise Architecture) (Zachman, 1987), vise à améliorer la réactivité du SI afin de soutenir efficacement cette stratégie. Pour ce faire, on cherche à aligner (Henderson et al., 1993) les processus métier de l'entreprise avec les architectures (applicative, fonctionnelle et technique) de son SI (espérant ainsi faciliter l'évolution conjointe du second par rapport au premier) (Wegmann, 2003).

Le cadre des entreprises orientées service (SOE) (Cauvet et al., 2008) a vocation à faciliter ce travail d'alignement, en rapprochant les tâches des processus métier avec des services orientés client. L'alignement est principalement réalisé entre les processus métier et l'architecture applicative de l'entreprise. Nous proposons dans cet article des règles d'urbanisation fonctionnelle visant à clarifier le processus de conception d'une architecture fonctionnelle, à permettre l'alignement de cette architecture avec les processus métier de l'entreprise, détecter des incohérences dans les processus métier et guider leur évolution pour un meilleur alignement avec le SI. Le choix de l'alignement de l'architecture fonctionnelle de l'entreprise avec les processus métier améliore la qualité de la conception des éléments applicatifs à réutiliser dans le SI, telle que décrite dans le cadre du SOEA (Service Oriented Enterprise Architecture) (Grigoriu, 2007).

Dans une entreprise orientée services, un processus métier est réalisé par un ou plusieurs services d'entreprise. Plus le nombre de services d'entreprise permettant de réaliser un processus métier est élevé, plus le coût dû à l'orchestration de ces services permettant de réaliser le processus métier dans sa globalité est élevé. Ce coût de l'orchestration fait apparaitre la nécessité d'avoir le meilleur alignement possible entre le processus métier à réaliser et la vue fonctionnelle cible du SI. Les tâches des processus de l'entreprise sont automatisées ou non dans son SI. L'alignement du SI sur l'organisation de l'entreprise devient un indicateur pertinent de la durabilité du SI par rapport à celle de l'entreprise. La durabilité de l'entreprise est mise en valeur par l'intégration de sa stratégie dans les processus de l'entreprise qu'elle utilise pour produire de la valeur. Un SI durable est mis en valeur par l'intégration de cette stratégie dans les systèmes du SI de l'entreprise qu'elle utilise pour produire de la valeur. 
La durabilité des grandes fonctions de l'entreprise (fonction stratégique, fonction opérationnelle, fonction logistique) est définie par l'aptitude de l'entreprise à évoluer au gré des transformations du marché, de l'évolution des technologies ou des fusions d'entreprise. Les technologies de l'information sont un catalyseur important de cette durabilité. Il est intéressant de noter que la mise en œuvre de ces technologies de l'information dans la réingénierie est de nature inductive et non déductive (Hammer et al., 1993). Ceci signifie que la détection d'une solution permet de résoudre des problèmes postérieurs à la solution. C'est un fondement de l'EA mis en œuvre lors de la définition d'un SI cible. Un but de l'EA est en effet, à partir d'une cartographie du SI de l'entreprise, de définir un SI cible permettant de supporter les évolutions du marché avant que ces évolutions ne soient définies de façon détaillée. La stratégie de l'entreprise prise en compte en entrée de la définition du SI cible a pour objet de décrire de façon macroscopique certaines de ces évolutions. C'est donc à partir de cette stratégie de l'entreprise que la cible de l'EA est définie.

Cet alignement est particulièrement significatif dans le cas d'une architecture SOA. L'intérêt de l'architecture orientée services (SOA - Service Oriented Architecture) est de permettre l'implémentation d'enchaînements de services réalisant un processus métier (Burlton, 2001). L'architecture orientée services fournit une méthodologie permettant d'organiser et d'utiliser des ressources du système informatique de manière distribuée (Schulte et al., 1996). L'architecture SOA peut ainsi favoriser la réutilisation des services offerts dans le SI de l'entreprise lors du développement de tout nouveau système. La difficulté est néanmoins de bien définir ces services afin de les réutiliser de la manière la plus pertinente, en particulier par rapport aux fonctions que réalisent ces services.

Lorsque l'alignement est imparfait, cette notion de coût, devient techniquement significative. L'orchestration de nombreux services fonctionnels au niveau du bus induit en effet des flux de données importants (Robinson, 2004). L'accessibilité des données à partir du bus de services nécessite de même une attention quant à leur protection, en particulier en ce qui concerne les données patrimoniales. Ces données représentant le patrimoine informationnel de l'entreprise sont en effet stratégiques.

Cet article propose une démarche de conception fonctionnelle des services d'entreprise basée sur l'alignement entre le processus métier à réaliser et une EA fonctionnelle du SI cible. La diminution du coût de développement est liée dans notre démarche à la prise en compte de l'EA et à l'étude d'un alignement entre le processus métier et l'EA fonctionnelle cible. L'autre apport concernant la sécurité des données patrimoniales est dû à une découpe en couches de l'EA fonctionnelle cible du SI. Cette découpe facilite en effet une conception des données pertinentes quant à la protection de leur accès.

La section suivante est consacrée à la définition des concepts permettant de décrire un processus métier et une EA fonctionnelle cible. La section 3 propose une démarche de conception de l'EA fonctionnelle cible fondée sur une architecture fonctionnelle en couches. Notre démarche de conception des services d'entreprise est décrite dans la section 4 . Son application à un des processus métier d'un opérateur de 
télécommunications est illustrée dans la section 5. Une conclusion sur le bénéfice de cette démarche termine l'article.

\section{Méta-modélisation et vues associées à l'EA}

Parmi les cadres de l'EA, celui de l'urbanisme permet de prendre en compte la modélisation (Salinesi et al., 2008). La description des concepts de chaque vue associée à l'urbanisme est ainsi faite dans un méta-modèle. Dans cette section, nous présentons uniquement les concepts des vues métier et fonctionnelles, les seules qui sont pertinentes par rapport à la démarche que nous présentons dans l'article. La méta-modélisation permet de modéliser les concepts décrivant les vues associées au cadre de l'urbanisme ainsi que leurs liens. L'intérêt de ce méta-modèle est que chaque modèle d'architecture du SI ou d'un système du SI est une instanciation du méta-modèle (Kleppe et al., 2003). L'objectif de réutilisation d'éléments du SI du niveau modèle est ainsi facilité par le partage, au niveau méta-modèle, des concepts permettant de décrire ces éléments.

\subsection{Définition des vues}

Les vues associées au cadre de l'urbanisme sont les vues d'architecture d'un système (fonctionnelle, technique, applicative) ${ }^{1}$ auxquelles la vue métier supportée par le SI est ajoutée. L'ensemble des vues recouvre les concepts utiles à la description d'un SI et de ses systèmes (Longépé, 2001) :

- une description du métier de l'entreprise dans la vue métier,

- une description des fonctions du SI dans la vue fonctionnelle,

- une description des technologies de déploiement des applications du SI dans la vue technique,

- une description des applications du SI dans la vue applicative.

Par rapport au développement d'un système du SI, la vue métier indique le "Pourquoi ?" de ce développement, la vue fonctionnelle précise le "Quoi ?", la vue technique décrit le "Avec quoi ?" et la vue applicative spécifie le "Comment ?".

Si l'on prend une analogie simpliste avec un processus de la vue métier lié à l'usage d'un logement, celui-ci serait supporté :

1. Par rapport au modèle des vues dit "4+1" (Kruchten, 1995) :

- la vue fonctionnelle correspond à la vue des scénarios et à la vue logique,

- la vue technique correspond à la vue physique,

- la vue applicative correspond à la vue développement et à la vue processus. 
- par une vue d'architecture fonctionnelle recouvrant les fonctions (se laver et se coucher), et,

- par une vue d'architecture technique avec l'infrastructure choisie (salle de bains, chambre à coucher).

La vue d'architecture applicative du système de type SOA représentant le logement recouvrirait :

- la réalisation sous forme de service de ces fonctions intégrant un déploiement sur cette infrastructure (se laver dans la salle de bains et se coucher dans la chambre).

\subsection{Vue métier}

La vue métier décrit les processus du cœur de métier de l'entreprise et leur structuration par rapport à l'organisation de l'entreprise. Les concepts de la vue métier décrits dans le Tableau 1 sont dérivés des notions UML d'évènement, de processus, de tâche, d'acteur ou de classe (Booch et al., 1999).

\begin{tabular}{|l|l|}
\hline \multicolumn{1}{|c|}{ Concept } & \multicolumn{1}{c|}{ Définition } \\
\hline Évènement métier & $\begin{array}{l}\text { Notification par un processus métier d'une information à un } \\
\text { autre processus métier, ou événement émis ou reçu par un } \\
\text { acteur métier ou un collaborateur métier. Sa signification est } \\
\text { directement perceptible par les différents acteurs individuels } \\
\text { de l'entreprise. Il peut être porteur d'informations ou de } \\
\text { matière. }\end{array}$ \\
\hline Processus métier & $\begin{array}{l}\text { Séquence de tâches réalisée par l'entreprise qui produit un } \\
\text { résultat dont la valeur est perceptible et mesurable pour un } \\
\text { acteur individuel lié à l'entreprise. Il doit être défini } \\
\text { indépendamment de toute organisation et de tout système } \\
\text { existant dans l'entreprise. }\end{array}$ \\
\hline Tâche métier & $\begin{array}{l}\text { Partie de traitement réalisée dans un processus métier, qui } \\
\text { d'une part est définie par les informations (entités métier) } \\
\text { qu'elle utilise en entrée et qu'elle produit en sortie, par les } \\
\text { événements métier reçus et produits, et qui d'autre part } \\
\text { respecte certaines règes : la tâche métier ne met en ouvre } \\
\text { qu'une action principale, elle n'est pas interruptible. Chaque } \\
\text { tâche est réalisée par un collaborateur métier. }\end{array}$ \\
\hline $\begin{array}{l}\text { Succession de } \\
\text { tâches métier }\end{array}$ & $\begin{array}{l}\text { Lien entre tâches métier indiquant un ordre de succession } \\
\text { des tâches d'un processus métier. Ce lien intègre les } \\
\text { contraintes associées à la succession (transition } \\
\text { conditionnelle, parallélisme et synchronisation de tâches). }\end{array}$ \\
\hline
\end{tabular}




\begin{tabular}{|l|l|}
\hline $\begin{array}{l}\text { Collaborateur } \\
\text { métier }\end{array}$ & $\begin{array}{l}\text { Représentation d'un rôle ou d'un ensemble de rôles joué } \\
\text { dans la réalisation des processus métier par un individu ou } \\
\text { une unité organisationnelle, interne au domaine modélisé } \\
\text { (par exemple, toute l'entreprise). Un collaborateur métier } \\
\text { contribue à la réalisation d'un ou plusieurs processus métier } \\
\text { (il a un rôle pour chaque processus métier auquel il } \\
\text { contribue), et plusieurs collaborateurs métier peuvent } \\
\text { contribuer à la réalisation d'un processus métier. Un } \\
\text { collaborateur métier émet ou reçoit des événements métier. }\end{array}$ \\
\hline Donnée métier & $\begin{array}{l}\text { Objet utilisé ou produit par les tâches métier. Une donnée } \\
\text { métier est produite par une seule tâche métier. }\end{array}$ \\
\hline
\end{tabular}

Tableau 1. Concepts de la vue métier

La production par un processus métier d'une valeur ajoutée au client est traduite par le fait que l'évènement métier déclencheur d'un processus est émis par le client du processus et que l'évènement métier traduisant la valeur ajoutée est reçu par ce même client.

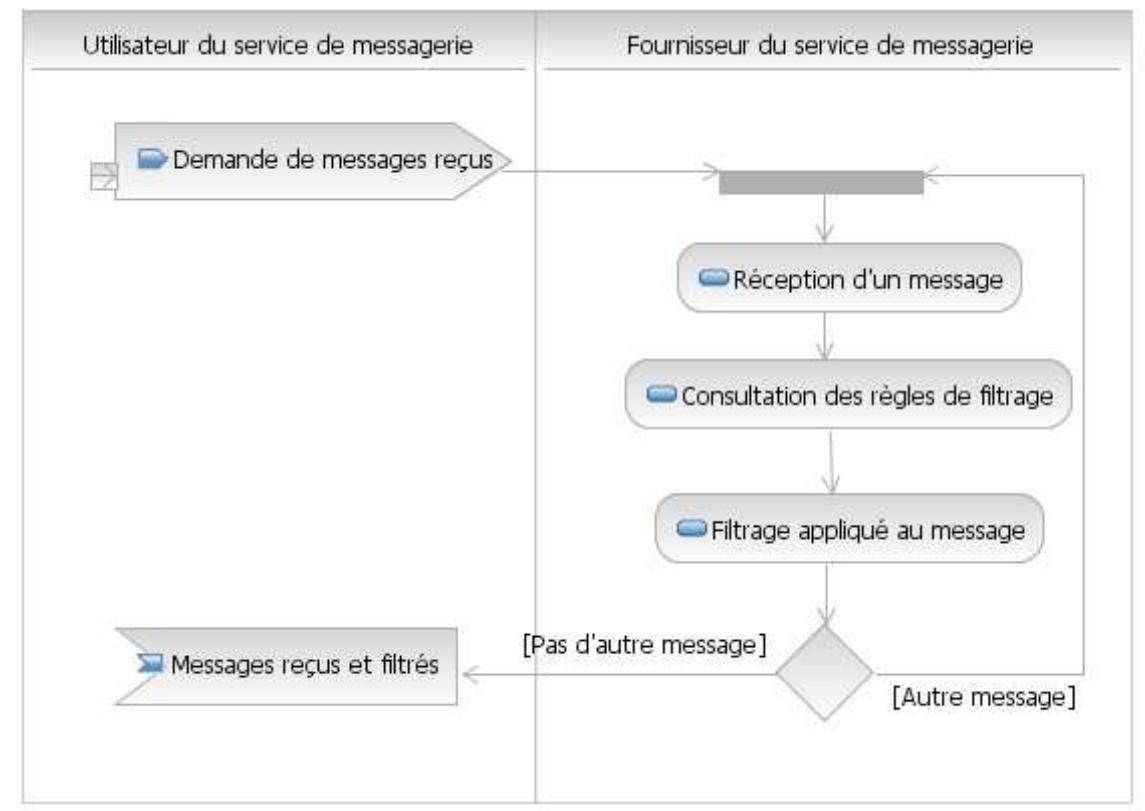

Figure 1. Illustration d'une vue métier 
Exemple : le méta-modèle de la vue métier est illustré dans la Figure 1 par le processus métier de réception d'un message électronique. Le client de l'entreprise est ici l'Utilisateur du service de messagerie qui est donc un collaborateur métier. La tâche métier Consultation des règles de filtrage succède à la tâche Réception d'un message qui est activée suite à l'émission de l'évènement métier Demande de messages reçus.

\subsection{Vue fonctionnelle}

Les concepts de la vue fonctionnelle sont définis dans le Tableau 2 à partir :

- des termes UML de dépendance, de classes et de relation entre classes (Booch et al., 1999) sont appliqués à la vue fonctionnelle,

- des termes d'îlot, de parcelle et de voie issus du PLU (Plan Local d'Urbanisme) (Code de l'urbanisme, 2008).

\begin{tabular}{|l|l|}
\hline \multicolumn{1}{|c|}{ Concept } & \multicolumn{1}{|c|}{ Définition } \\
\hline Îlot fonctionnel & $\begin{array}{l}\text { Regroupement de services fonctionnels qui offrent une vue } \\
\text { externe de l'ilot. }\end{array}$ \\
\hline Type îlot fonctionnel & $\begin{array}{l}\text { Le typage d'un îlot fonctionnel permet la localisation de } \\
\text { l'élément sur une couche de la vue fonctionnelle. }\end{array}$ \\
\hline $\begin{array}{l}\text { Dépendance entre } \\
\text { îlots fonctionnels }\end{array}$ & $\begin{array}{l}\text { Voie orientée entre îlots fonctionnels représentant un } \\
\text { ensemble de services fournis par l'îlot cible de la } \\
\text { dépendance à l'îlot à l'origine de la dépendance. }\end{array}$ \\
\hline Donnée fonctionnelle & $\begin{array}{l}\text { Objet utilisé ou produit par les îlots fonctionnels. Une } \\
\text { donnée fonctionnelle est produite par un seul îlot } \\
\text { fonctionnel. }\end{array}$ \\
\hline Service fonctionnel & $\begin{array}{l}\text { Parcelle d'un îlot représentant sa vue externe. Il offre un } \\
\text { accès à des données fonctionnelles ou à des traitements sur } \\
\text { ces données (paramètres de sortie) à partir d'autres données } \\
\text { fonctionnelles (paramètres d'entrée). }\end{array}$ \\
\hline
\end{tabular}

Tableau 2. Concepts de la vue fonctionnelle

Un service d'entreprise est conçu à partir d'un assemblage de services fonctionnels et de dépendances entre îlots fonctionnels fournissant ces services. Les services fonctionnels et les dépendances entre îlots fonctionnels doivent être conformes à l'EA fonctionnelle cible.

Définition : La vue fonctionnelle d'un service d'entreprise est une séquence de services fonctionnels réalisant un ensemble de tâches métier se succédant dans un processus métier. 
Exemple : quelques concepts du méta-modèle de la vue fonctionnelle sont décrits dans la Figure 2 par des îlots ayant trait à la messagerie électronique : Réception d'un message et Sécurité de la messagerie.

L'îlot fonctionnel Réception d'un message fournit un service fonctionnel Recevoir un message à lîlot fonctionnel Sécurité de la messagerie, qui donc en dépend. Ce service fonctionnel pourrait avoir pour paramètre de sortie une date de réception de message. Le concept de typage d'un îlot fonctionnel est la base de la conception des îlots de l'EA fonctionnelle cible décrite dans le paragraphe suivant.

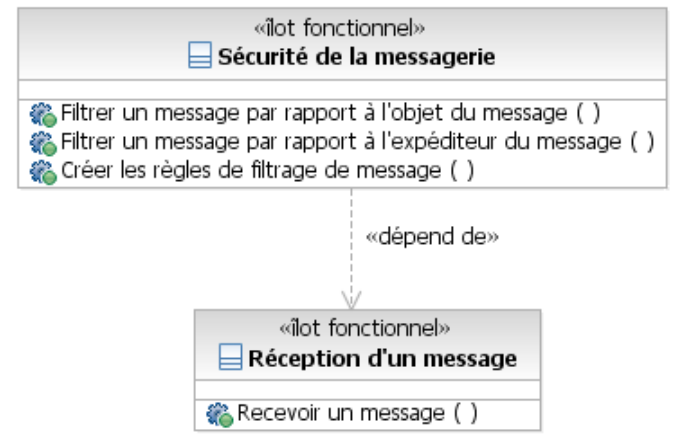

Figure 2. Illustration d'une vue fonctionnelle

La vue fonctionnelle d'un service d'entreprise pourrait être la séquence constituée du service Filtrer un message par rapport à l'objet du message et du service Recevoir un message qui réalise les tâches métier Filtrage appliqué au message et Réception d'un message.

\section{Intérêt du typage pour la conception de l'EA fonctionnelle cible}

La conception des îlots du PLU fonctionnel d'un SI est une activité complexe de l'urbanisation. La complexité nait de l'intégration concrète de la stratégie de l'entreprise dans la vue fonctionnelle d'un SI qui est abstraite. Afin d'assister cette conception, nous proposons une solution fondée sur le typage de chaque îlot fonctionnel par rapport à la durée de vie de son instance. Un modèle type des dépendances entre des îlots de type différent permet l'atteinte de l'objectif.

\subsection{Définition du typage d'un îlot fonctionnel}

Le principe du typage est issu du concept de couche architecturale inspiré du modèle OSI (Open Systems Interconnect) (Zimmermann, 1980). Le typage de 
chaque élément permet la localisation de l'élément sur une couche. Ce principe est appliqué à la conception des îlots fonctionnels et des dépendances qui les relient.

Le principe de ce typage est de considérer la dépendance de la durée de vie d'une instance au sens UML du service fonctionnel par rapport à celle du processus supporté.

Définition : un îlot fonctionnel est de type

- flux si la durée de vie d'une instance d'au moins un de ses services fonctionnels est dépendant du processus supporté ;

- stock si la durée de vie d'une instance d'au moins un de ses services fonctionnels est indépendante du processus supporté.

Soit $\mathrm{I}_{\mathrm{f}}$ l'ensemble des îlots fonctionnels du PLU, soit $\mathrm{T}$ l'ensemble des types d'îlots fonctionnels, alors le typage $t$ d'un l'îlot fonctionnel $i$ peut être représenté comme une fonction de $\mathrm{I}_{\mathrm{f}}$ vers $\mathrm{P}(\mathrm{T})$, où $\mathrm{P}(\mathrm{T})$ désigne l'ensemble des parties de $\mathrm{T}$ :

$$
\begin{aligned}
& t: I_{f} \rightarrow P(T) \\
& i \mapsto t ; t \subset P(\{\text { flux, stock }\})
\end{aligned}
$$

Un îlot peut donc être de type \{flux, stock\}.

Exemple : le processus supporté Figure 1 est celui de l'usage d'une messagerie électronique, l'îlot fonctionnel Sécurité de la messagerie est de type flux pour les services fonctionnels Filtrer un message par rapport à l'objet du message, Filtrer le message par rapport à l'expéditeur du message car toutes instances de ces services fonctionnels ont une durée de vie dépendante du processus d'usage d'une messagerie électronique. L'îlot fonctionnel sécurité de la messagerie est de type stock pour son services fonctionnel Créer les règles de filtrage de message. La création de règles de filtrage des messages indésirables est en effet transverse aux différents usages d'un service de messagerie électronique. Le cycle de vie des instances de ce service fonctionnel est donc indépendant de celui de l'usage du service. L'îlot fonctionnel Réception d'un message défini par le service fonctionnel Recevoir un message est de type flux puisque ce service fonctionnel a un cycle de vie dépendant de l'usage d'un service de messagerie.

D'après l'équation [1], la formulation de ce typage est la suivante :

$$
\begin{aligned}
& t(\text { Sécurité de la messagerie })=\{\text { Flux }, \text { Stock }\} \\
& t(\text { Réception d'un message })=\{\text { Flux }\}
\end{aligned}
$$

Idéalement, le typage des îlots fonctionnels doit permettre d'associer chaque îlot à un type flux ou à un type stock de l'architecture fonctionnelle d'un système. Afin de stabiliser les dépendances entre îlots, nous proposons la règle d'urbanisation dite Règle d'urbanisme - Flux et stock qui contraint l'orientation des dépendances. 
Règle d'urbanisme - Flux et stock : si un îlot fonctionnel de type flux et un îlot fonctionnel de type stock sont dépendants, alors l'îlot fonctionnel de type flux dépend de l'îlot fonctionnel de type stock.

La raison de l'orientation de cette dépendance est que chaque processus est d'abord supporté par un îlot fonctionnel de type flux. Dans l'illustration, le processus de réception d'un message est réalisé dans un premier temps par l'îlot fonctionnel Réception d'un message. Pour réaliser le processus, cet îlot fonctionnel a besoin d'îlots fonctionnels transverses à cette réception, tels que la gestion de certaines règles de filtrage. Cette gestion est sous la responsabilité de l'îlot fonctionnel de type stock de gestion des règles de filtrage. Le besoin de cet îlot transverse se traduit par la dépendance de l'îlot fonctionnel de type flux de Réception d'un message vis-à-vis de l'îlot fonctionnel de type stock de gestion des règles de filtrage.

Il est utile de traduire cette dépendance au niveau des données produites par chacun de ces îlots. Le besoin d'un îlot de type stock par un îlot de type flux pour réaliser un processus est illustré par la dépendance de la donnée caractérisant la réception d'un message, telle que sa date de réception, par rapport à la donnée caractérisant une règle de filtrage. Cette dépendance de données permet de sécuriser les données produites par l'îlot fonctionnel de type stock. Le principe est en effet de réaliser cet îlot de type stock avec des exigences de sécurité élevées (dans l'exemple: contrôle d'accès suivant le rôle de l'utilisateur tel qu'administrateur, développeur du service de messagerie et suivant la donnée) alors que la réalisation de l'îlot fonctionnel de type flux est associée à des exigences de sécurité plus faible (dans l'exemple : authentification de l'utilisateur du service de messagerie).

\subsection{Transformation des îlots fonctionnels contrainte par le typage}

Dans cette section, nous revenons sur le cas où des îlots possèdent des services fonctionnels de type différents. Dans ce cas, l'îlot est doublement typé \{flux, stock\}, nous proposons alors de le scinder en différents ilots. Nous décrivons ici la manière de procéder et indiquons l'évolution des dépendances qui en découlent.

La fonction s définie ci-après représente la scission d'un îlot fonctionnel.

Définition : Si T est l'ensemble des types d'îlots fonctionnels, si $\mathrm{I}_{\mathrm{f}}$ est l'ensemble des îlots fonctionnels du PLU et si $\mathrm{P}\left(\mathrm{I}_{\mathrm{f}} \mathrm{x}\right.$ T) est l'ensemble des parties de $\mathrm{I}_{\mathrm{f}} \mathrm{x}$ T, c'està-dire l'ensemble des sous-ensembles de $\mathrm{I}_{\mathrm{f}} \mathrm{x} \mathrm{T}$, alors la scission $s$ de l'îlot fonctionnel $i$ en $n$ îlots fonctionnels, dont $i_{1}$ de type $t_{1}$ et $i_{n}$ de type $t_{n}$, peut être représentée de la manière suivante :

$$
\begin{aligned}
& s: I_{f} \rightarrow P\left(I_{f} \times T\right) \\
& i \mapsto\left\{\left(i_{1}, t_{1}\right), \ldots,\left(i_{n}, t_{n}\right)\right\}, t\left(i_{1}\right)=\left\{t_{1}\right\}, \ldots, t\left(i_{n}\right)=\left\{t_{n}\right\}
\end{aligned}
$$


Les services fonctionnels d'un même îlot sont fortement couplés. Ce couplage est représenté fonctionnellement par une dépendance entre les îlots issus de la scission. Le modèle des dépendances issues de la scission d'îlots fonctionnels doit être conforme à la règle de dépendance entre îlots fonctionnels suivant leur type défini dans la Règle d'urbanisme - Flux et stock.

Exemple : dans l'illustration choisie de sécurité liée à la réception d'un message, si l'îlot fonctionnel Sécurité de la messagerie est à la fois du type flux et du type stock, alors cet îlot doit être scindé en deux îlots fonctionnels :

- Sécurité de la messagerie - Filtrage de message de type flux défini par les services fonctionnels Filtrer un message par rapport à l'objet du message et Filtrer le message par rapport à l'expéditeur du message,

- Sécurité de la messagerie - Gérer les règles de filtrage de type stock défini par le service fonctionnel Créer les règles de filtrage de message.

Ceci est équivalent, d'après [2] à :

$s($ Sécurité de la messagerie $)=\left\{\begin{array}{l}(\text { Sécurité de la messagerie filtrage de message, Flux }), \\ (\text { Sécurité de la messagerie gérer les règles de filtrage, Stock })\end{array}\right\}$

D'après la Règle d'urbanisme - Flux et stock, le nouvel îlot fonctionnel Sécurité de la messagerie - Filtrage de message dépend de l'îlot fonctionnel Sécurité de la messagerie - Gérer les règles de filtrage. La dépendance de ces deux îlots fonctionnels est représentée dans la Figure 3. Dans ce diagramme de classes UML, le type d'un îlot est inséré dans le stéréotype de la classe UML de l'îlot. Par exemple, le stéréotype de l'îlot fonctionnel Sécurité de la messagerie - Filtrage de message est "îlot fonctionnel - flux".

\subsection{Transformation des dépendances entre îlots contrainte par le typage des îlots}

Lors d'une scission d'îlot fonctionnel, la règle d'urbanisme suivante permet de transformer les dépendances ayant pour source ou pour cible l'îlot scindé.

Règle d'urbanisme - Scission îlot multi-typé : lors de la scission d'un îlot fonctionnel, sous réserve de la Règle d'urbanisme - Flux et stock :

- toutes les dépendances ayant pour source l'îlot fonctionnel scindé sont transformées en dépendances ayant pour source les îlots fonctionnels issus de la scission ;

- toutes les dépendances ayant pour cible l'îlot fonctionnel scindé sont transformées en dépendances ayant pour cible les îlots fonctionnels issus de la scission.

Exemple : dans le diagramme de classes de la Figure 3, la dépendance avec l'îlot fonctionnel Réception d'un message est transformée en : 
- une dépendance de l'îlot fonctionnel Sécurité de la messagerie - Filtrage de message de type flux vers l'îlot fonctionnel Réception d'un message de type flux qui satisfait la Règle d'urbanisme - Flux et stock.

La dépendance de l'îlot fonctionnel Sécurité de la messagerie - Gérer les règles de filtrage de type stock vers l'îlot fonctionnel Réception d'un message de type flux ne peut être créée conformément à la Règle d'urbanisme - Scission îlot multi-typé car elle ne respecte pas la Règle d'urbanisme - Flux et stock.

Il est intéressant de noter que l'absence de report de cette dépendance dans le modèle d'îlots fonctionnels a un sens métier. La gestion des règles de filtrage de message est en effet indépendante de la réception d'un message.

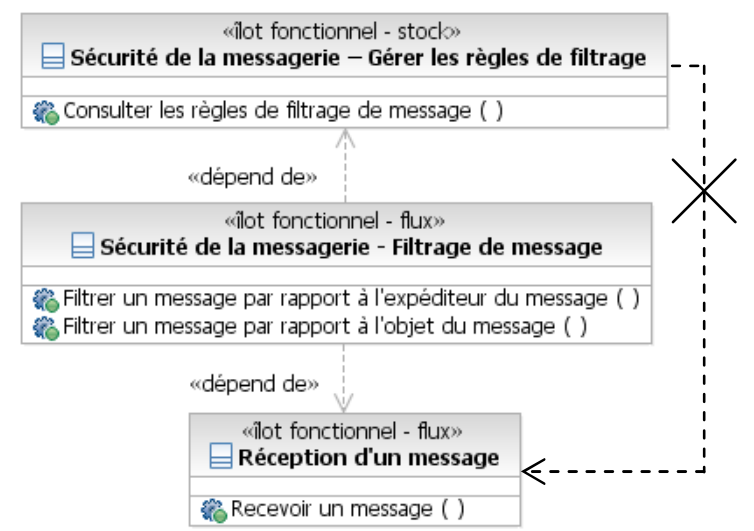

Figure 3. Illustration des îlots et des dépendances fonctionnels respectant la contrainte par typage des îlots

\section{Conception de services d'entreprise par alignement avec l'EA fonctionnelle cible}

Dans cette section, nous proposons une démarche de conception de services d'entreprise. Elle recouvre les activités suivantes :

- L'alignement statique des tâches d'un processus métier par rapport à l'EA fonctionnelle cible fait par un expert ;

- L'alignement dynamique automatisé des tâches d'un processus métier, soit leur ordre de succession dans le déroulement du processus, par rapport à l'EA fonctionnelle cible ;

- Un bilan de l'alignement permettant au concepteur des services d'entreprise d'améliorer le descriptif du processus métier par rapport à l'EA fonctionnelle cible ; 
- La conception fonctionnelle automatique de services d'entreprise réalisant le processus métier.

L'alignement d'un processus métier par rapport à l'EA fonctionnelle cible nécessite de définir les critères d'alignement. L'intérêt du méta-modèle des vues du cadre de l'urbanisme est de proposer des concepts décrivant chaque vue. A partir du métamodèle introduit en section 2 , nous proposons donc le critère d'alignement suivant consistant à associer des concepts de la vue métier à des concepts de la vue fonctionnelle et à vérifier leur cohérence.

Les concepts de donnée métier et de donnée fonctionnelle auraient pu aussi être retenus pour définir l'alignement. Or, d'après leur définition, l'alignement de ces données est équivalent à l'alignement de la tâche qui produit la donnée métier et du service fonctionnel, ou des services fonctionnels, fournis par l'îlot fonctionnel qui produit la donnée fonctionnelle.

\subsection{Alignement statique d'un processus métier par rapport à l'EA fonctionnelle cible}

Dans cette section nous définissons l'alignement statique dans le méta-modèle de la Figure 4. Il cible les concepts de tâche métier et de service fonctionnel appartenant respectivement aux vues métier et fonctionnelle.

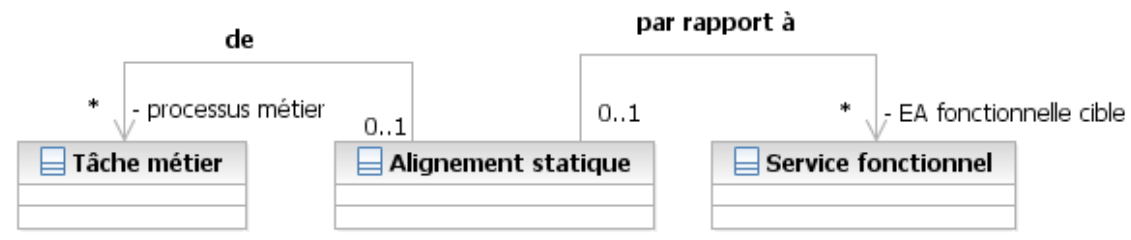

Figure 4. Méta-modèle de l'alignement statique d'une vue métier et d'une vue fonctionnelle

Définition : L'alignement statique d'une vue métier par rapport à une vue fonctionnelle est défini de la façon suivante. Une tâche métier est alignée avec un ou plusieurs services fonctionnels

- si la tâche métier a un sens partagé avec au moins un service fonctionnel,

- et si tous les services fonctionnels alignés avec une même tâche métier doivent être couverts par un graphe de dépendance connexe.

Cette dernière condition signifie que les îlots fonctionnels, qui fournissent les services fonctionnels alignés avec une même tâche, et leurs dépendances forment un graphe connexe. 
Exemple : l'alignement statique est illustré par celui du processus de réception d'un message décrit Figure 1 par rapport à l'EA fonctionnelle cible décrite Figure 3 :

- la tâche métier Réception d'un message est alignée avec le service fonctionnel Recevoir un message,

- la tâche métier Consultation des règles de filtrage est alignée avec le service fonctionnel Consulter les règles de filtrage de message,

- la tâche métier Filtrage appliqué au message est alignée avec les services fonctionnels Filtrer un message par rapport à l'expéditeur du message et Filtrer un message par rapport à l'objet du message.

La condition de graphe de dépendance connexe est remplie dans ce dernier alignement puisque les services sont fournis par le même îlot fonctionnel.

\subsection{Alignement dynamique d'un processus métier par rapport à l'EA fonctionnelle cible}

L'alignement dynamique décrit dans le méta-modèle de la Figure 5 est défini par l'association entre une relation de succession d'activités métier et une relation de dépendance de services fonctionnels.

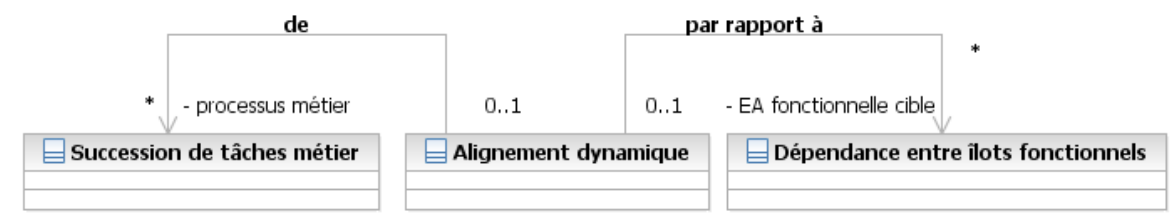

Figure 5. Méta-modèle de l'alignement dynamique d'une vue métier et d'une vue fonctionnelle

Définition : L'alignement dynamique d'une vue métier par rapport à une vue fonctionnelle est défini de la façon suivante. Une succession de 2 tâches métier, $\mathrm{T}_{1}$ et $T_{2}$ telle que $T_{2}$ succède à $T_{1}$, est alignée avec une vue fonctionnelle si

- il existe au moins un service fonctionnel $S_{1}$ aligné statiquement avec $T_{1}$,

- il existe au moins un service fonctionnel $\mathrm{S}_{2}$ aligné statiquement avec $\mathrm{T}_{2}$,

- tels que l'îlot fonctionnel fournisseur de $S_{2}$ dépende de lîlot fonctionnel fournisseur de $S_{1}$.

Du fait de sa définition, l'alignement dynamique est une étape automatisable qui vient compléter l'alignement statique.

Exemple : l'alignement dynamique est illustré à partir du processus de réception d'un message décrit Figure 1 par rapport à l'EA fonctionnelle cible décrite Figure 3 : 
- la succession de la tâche métier Réception d'un message par la tâche métier Consultation des règles de filtrage n'est pas alignée avec la vue fonctionnelle car il n'existe pas de dépendance de l'îlot fonctionnel Sécurité de la messagerie - Gérer les règles de filtrage (îlot fournisseur du service Consulter les règles de filtrage de message aligné avec la tâche Consultation des règles de filtrage) vers l'îlot fonctionnel Réception d'un message (îlot fournisseur du service Recevoir un message aligné avec la tâche Réception d'un message),

- la succession de la tâche métier Consultation des règles de filtrage par la tâche métier Filtrage appliqué au message est aligné avec la dépendance de l'îlot fonctionnel Sécurité de la messagerie - Filtrage de message (îlot fournisseur des services Filtrer un message par rapport à l'expéditeur du message et Filtrer un message par rapport à l'objet du message alignés avec la tâche Filtrage appliqué au message) vis-à-vis de l'îlot fonctionnel Sécurité de la messagerie - Gérer les règles de filtrage (îlot fournisseur du service Consulter les règles de filtrage de message aligné avec la tâche Consultation des règles de filtrage).

\subsection{Bilan de l'alignement et correction d'un processus métier par rapport à l'EA fonctionnelle cible}

Le bilan de l'alignement d'un processus métier par rapport à l'EA fonctionnelle permet au concepteur des services d'entreprise de corriger le processus métier à réaliser.

4.3.1. Axiomatisation de l'alignement d'un processus métier par rapport à l'EA fonctionnelle cible

Le comportement attendu de l'alignement des vues métier et fonctionnelle a pour paramètres les concepts définissant les alignements statique et dynamique. Une axiomatisation permet de décrire ce comportement attendu (Shepperd et al., 1993).

Nous proposons les quatre axiomes suivants pour décrire l'Alignement de la vue Métier par rapport à la vue Fonctionnelle (AMF). Ils ciblent les concepts de la vue métier définissant l'alignement.

AMF1 - Ajout d'une nouvelle tâche dans le processus métier à réaliser : l'alignement résultant de l'ajout d'une nouvelle tâche dans le processus métier à réaliser est, par rapport à l'alignement précédent,

- moins bon ou identique si la tâche ne peut être alignée statiquement avec au moins un service de l'EA fonctionnelle cible,

- meilleur si la tâche peut être alignée statiquement avec au moins un service de l'EA fonctionnelle cible. 
AMF2 - Ajout d'une relation de succession entre deux tâches du processus métier à réaliser : l'alignement résultant de l'ajout d'une relation de succession entre deux tâches du processus métier à réaliser est, par rapport à l'alignement précédent,

- moins bon ou identique s'il n'existe pas de relation de dépendance entre deux îlots de l'EA fonctionnelle cible alignée avec la relation de succession ajoutée,

- meilleur s'il existe au moins une relation de dépendance entre deux îlots de l'EA fonctionnelle cible alignée avec la relation de succession ajoutée.

AMF3 - Suppression d'une tâche dans le processus métier à réaliser : l'alignement résultant de la suppression d'une tâche dans le processus métier à réaliser est, par rapport à l'alignement précédent,

- moins bon si la tâche peut être alignée statiquement avec au moins un service de l'EA fonctionnelle cible,

- meilleur ou identique si la tâche ne peut être alignée statiquement avec au moins un service de l'EA fonctionnelle cible.

AMF4 - Suppression d'une relation de succession entre deux tâches du processus métier à réaliser : l'alignement résultant de la suppression d'une relation de succession entre deux tâches du processus métier à réaliser est, par rapport à l'alignement précédent,

- moins bon s'il existe au moins une relation de dépendance entre deux îlots de l'EA fonctionnelle cible alignée avec la relation de succession supprimée,

- meilleur ou identique s'il n'existe pas de relation de dépendance entre deux îlots de l'EA fonctionnelle cible alignée avec la relation de succession supprimée.

\subsubsection{Mesure de l'alignement d'un processus métier par rapport à l'EA fonctionnelle cible}

La mesure de l'alignement est un outil mis à la disposition du concepteur de services d'entreprise afin de vérifier la cohérence de son processus métier par rapport à l'EA fonctionnelle cible du SI. La mesure proposée est le produit du pourcentage de tâches métier alignées par le pourcentage de relations de successions entre tâche métier alignées.

Définition : La mesure MAMF de l'alignement d'un processus métier $\mathrm{P}$ par rapport à l'EA fonctionnelle cible est telle que :

$$
\operatorname{MAMF}(P)=\left(\frac{N_{t}(P)-N_{\text {tna }}(P)}{N_{t}(P)}\right) *\left(\frac{N_{s}(P)-N_{\text {sna }}(P)}{N_{s}(P)}\right)
$$

avec pour paramètres,

- le nombre $\mathrm{N}_{\mathrm{t}}(\mathrm{P})$ de tâches du processus métier $\mathrm{P}$ à réaliser, 
- le nombre $\mathrm{N}_{\text {tna }}(\mathrm{P})$ de tâches du processus métier $\mathrm{P}$ à réaliser non alignées avec l'EA fonctionnelle cible,

- le nombre $\mathrm{N}_{\mathrm{s}}(\mathrm{P})$ de relations de successions de tâches du processus métier $\mathrm{P}$ à réaliser,

- le nombre $\mathrm{N}_{\text {sna }}(\mathrm{P})$ de relations de successions de tâches du processus métier $\mathrm{P}$ à réaliser non alignées avec des relations de dépendances entre îlots fonctionnels de l'EA fonctionnelle cible.

La mesure MAMF prend toutes ses valeurs dans l'intervalle [0, 1]. MAMF vaut 0 lorsqu'aucune tâche ou aucune relation de succession de tâches du processus métier à réaliser ne sont alignées avec l'EA fonctionnelle cible. A l'opposé, MAMF vaut 1 lorsque l'alignement est parfait, c'est-à-dire lorsque toutes les tâches métier et leurs relations de succession sont alignées avec l'EA fonctionnelle cible.

La mesure MAMF vérifie les quatre axiomes AMF1, AMF2, AMF3, AMF4 de l'alignement fonctionnel par rapport à la vue métier. La démonstration n'est pas reproduite ici. Elle est analogue à celle faite pour la mesure de l'alignement de la vue fonctionnelle par rapport à la vue métier dans (Simonin, 2009).

Exemple : dans l'illustration choisie des alignements statique et dynamique, d'après [3] :

$$
\begin{aligned}
& N_{t}(P)=3, N_{\text {tna }}(P)=0, N_{s}(P)=2, N_{\text {sna }}(P)=1 \\
& \Rightarrow \operatorname{MAMF}(P)=\left(\frac{3-0}{3}\right) *\left(\frac{2-1}{2}\right)=\frac{1}{2}
\end{aligned}
$$

La mesure pourrait être améliorée en estimant que le processus métier n'a pas été correctement spécifié. Les tâches de réception de message et de consultation des règles de filtrage peuvent en effet être parallélisées (cf. Figure 6).

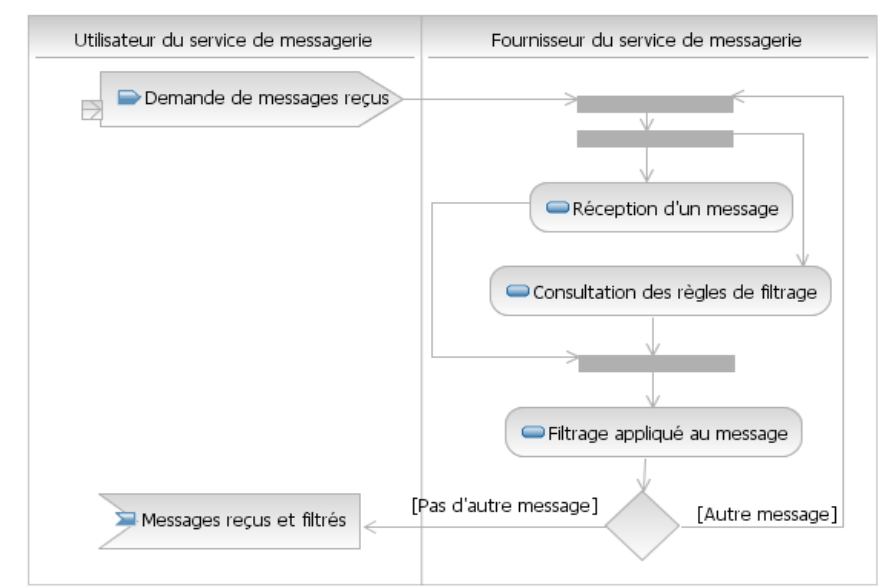

Figure 6. Illustration de l'alignement parfait d'un processus métier avec l'EA fonctionnelle cible 
Dans ce cas l'alignement est parfait puisque :

- la succession de la tâche métier Réception d'un message par la tâche métier Filtrage appliqué au message est alignée avec la dépendance de lîlot fonctionnel Sécurité de la messagerie - Filtrage de message (îlot fournisseur des services Filtrer un message par rapport à l'expéditeur du message et Filtrer un message par rapport à l'objet du message alignés avec la tâche Filtrage appliqué au message) vis-à-vis de l'îlot fonctionnel Réception d'un message (îlot fournisseur du service Recevoir un message aligné avec la tâche Réception d'un message),

- la succession de la tâche métier Consultation des règles de filtrage par la tâche métier Filtrage appliqué au message est aligné avec la dépendance de l'îlot fonctionnel Sécurité de la messagerie - Filtrage de message (îlot fournisseur des services Filtrer un message par rapport à l'expéditeur du message et Filtrer un message par rapport à l'objet du message alignés avec la tâche Filtrage appliqué au message) vis-à-vis de l'îlot fonctionnel Sécurité de la messagerie - Gérer les règles de filtrage (îlot fournisseur du service Consulter les règles de filtrage de message aligné avec la tâche Consultation des règles de filtrage).

\subsection{Conception fonctionnelle des services d'entreprise}

Suite à l'optimisation de l'alignement d'un processus métier à réaliser avec l'EA fonctionnelle cible, l'activité de conception fonctionnelle de services d'entreprise a pour objectif de générer automatiquement les services d'entreprise réalisant un processus métier. La conception fonctionnelle est ainsi la traduction du processus métier en une architecture fonctionnelle assurant l'intégration dans le SI.

\subsubsection{Règle de conception des services d'entreprise}

Une règle d'urbanisme utile à l'activité de conception fonctionnelle des services d'entreprise a pour objectif de protéger les données patrimoniales. Ces données sont en effet des données fonctionnelles produites par un îlot fonctionnel de type stock. Les attributs de ces données patrimoniales sont obligatoirement des paramètres de sortie des services fonctionnels offerts par ce type d'îlots.

Règle d'urbanisme - SOE piloté par le flux : tout service d'entreprise doit avoir, pour premier service fonctionnel de la séquence, un service fourni par un îlot fonctionnel de type flux.

Cette définition d'un service d'entreprise permet de n'accéder qu'à des îlots fonctionnels de type flux à partir du bus de services fonctionnels. De cette façon, les données de stock ne peuvent être accessibles directement à partir du bus et peuvent donc être protégées lors de la réalisation applicative des services d'entreprise. Cette règle facilitant la mise en œuvre de la sécurité liée aux données patrimoniales dans une entreprise orientée services est un sujet particulièrement sensible pour toutes entreprise offrant un SOE. 


\subsubsection{Conception automatique des services d'entreprise}

L'automatisation de la conception fonctionnelle des services d'entreprise est fondée sur l'approche MDA (Model Driven Architecture) (OMG - MDA, web). L'approche MDA est une norme proposée par l'OMG (Object Management Group) qui cible la transformation de modèles. Les modèles impactés sont le PIM (Platform Independent Model), le PSM (Platform Specific Model) et le modèle de la plateforme (Miller et al., 2003). L'utilisation méthodologique de ce modèle de plateforme sous le sigle de PDM (Platform Description Model) est définie par le fait que le PSM est une configuration du PIM pour un PDM donné (Gervais, 2002). Le PDM est dans notre approche une extension de la notion de PDM définie par l'OMG. L'originalité de l'utilisation de l'approche MDA est ici d'assimiler les modèles conçus par les urbanistes fonctionnels à des PDM. La raison est que le rôle du PDM définissant des contraintes lors d'une transformation de modèles prend tout son sens avec les modèles d'EA d'un SI cible.

L'idée représentée dans la Figure 7 est de transformer un modèle de processus métier, représenté par un PIM, en un modèle de services d'entreprise, représenté par

nn:

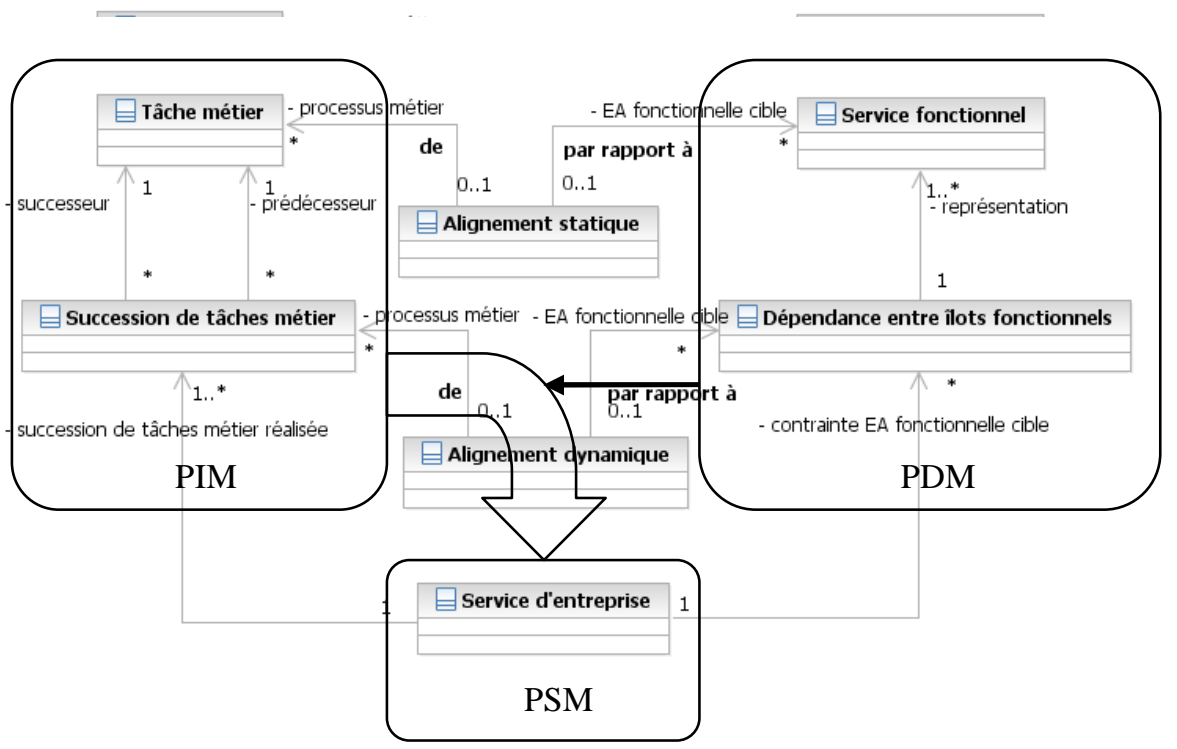

Figure 7. Méta-modélisation de l'activité de conception automatique de services d'entreprise

L'algorithme justifiant le caractère automatique de la transformation est fondé sur les principes suivants : 
- La séquence de services fonctionnels alignés sur le processus métier à réaliser débute avec le ou les services fonctionnels alignés avec la dernière tâche métier du processus et se termine avec le ou les services fonctionnels alignés avec la première tâche métier du processus ;

- De façon à satisfaire la Règle d'urbanisme - SOE piloté par le flux , la séquence de services fonctionnels est scindée automatiquement à chaque succession d'un service fourni par un îlot fonctionnel de type stock par un service fourni par un îlot fonctionnel de type flux ;

- Un service d'entreprise est une séquence de services fonctionnels compris entre deux scissions dont le premier est fourni par un îlot de type flux, et de dépendances entre les îlots fonctionnels qui les fournissent.

Dans ce cas d'alignement parfait, le service d'entreprise réalise complètement le processus métier.

Exemple : pour le processus métier de la Figure 8, la Règle d'urbanisme - SOE piloté par le flux est satisfaite pour les deux services d'entreprise entourés :

- Filtrage de message par rapport à l'expéditeur du message dépendant de règles de filtrage de message et de la réception d'un message

- Filtrage de message par rapport à l'objet du message dépendant de règles de filtrage de message et de la réception d'un message

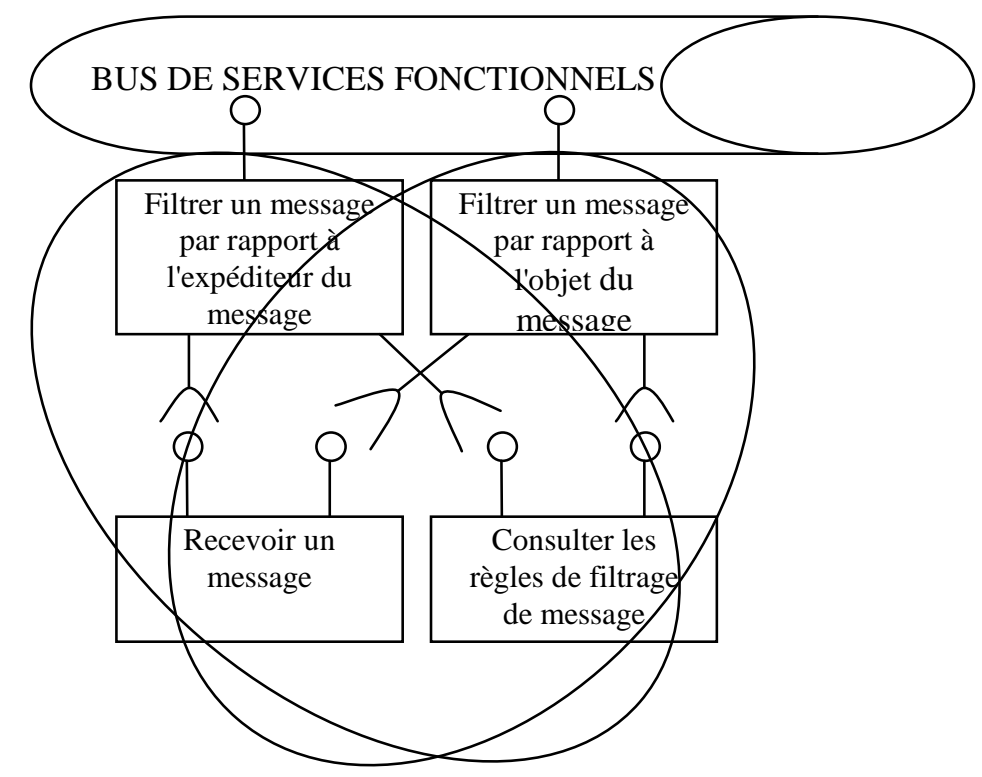

Figure 8. Illustration de l'activité de conception automatique de services d'entreprise à partir d'un alignement parfait du processus métier réalisé 
Pour ces deux services d'entreprise, les services fonctionnels accessibles directement à partir du bus sont en effet fournis par l'îlot fonctionnel de type flux, Sécurité de la messagerie - Filtrage de message.

Les règles de filtrage de message sont les données produites par l'îlot fonctionnel de type stock Sécurité de la messagerie - Gérer les règles de filtrage. Ces données patrimoniales à protéger ne sont pas accessibles directement par le bus de services fonctionnels. L'utilisation du bus de services fonctionnels est de plus optimisée puisque les données fonctionnelles produites par l'îlot fonctionnel Réception d'un message ne transitent plus sur le bus de services fonctionnels.

\section{Application : processus de complétion des offres au client}

L'application de la démarche de conception fonctionnelle de services d'entreprise cible un des processus métier décrits dans eTOM [eTOM, web] par le TMF (TeleManagementForum). Ce processus lié au domaine des télécommunications est celui de «complétion » des offres au client décrit dans la Figure 9.

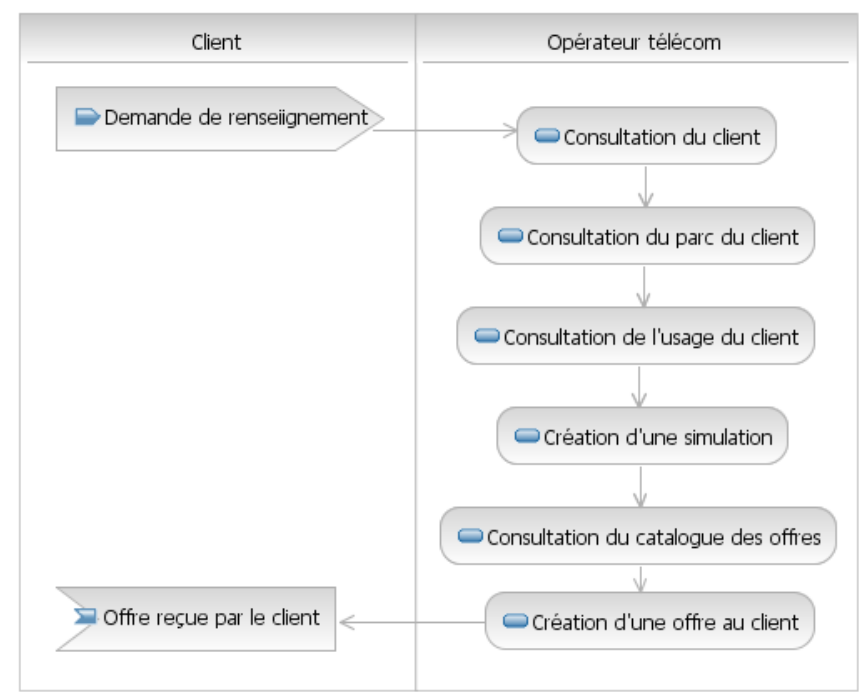

Figure 9. Processus métier de complétion des offres au client 
Ce processus métier de complétion des offres proposée à un client consiste à lui suggérer des offres déduites d'une simulation faite sur son usage des produits et services de son parc télécom. La valeur ajoutée vue du client est de compléter son parc de façon à satisfaire son usage de télécommunications. Un extrait de l'EA fonctionnelle cible d'un SI des offres, ou SI commercial, est représenté dans la Figure 10. Cette EA fonctionnelle cible conçue par les urbanistes intègre la mise en œuvre du typage de chaque îlot fonctionnel respectant les règles décrites dans le $\S 3$, en particulier la Règle d'urbanisme - Flux et stock.

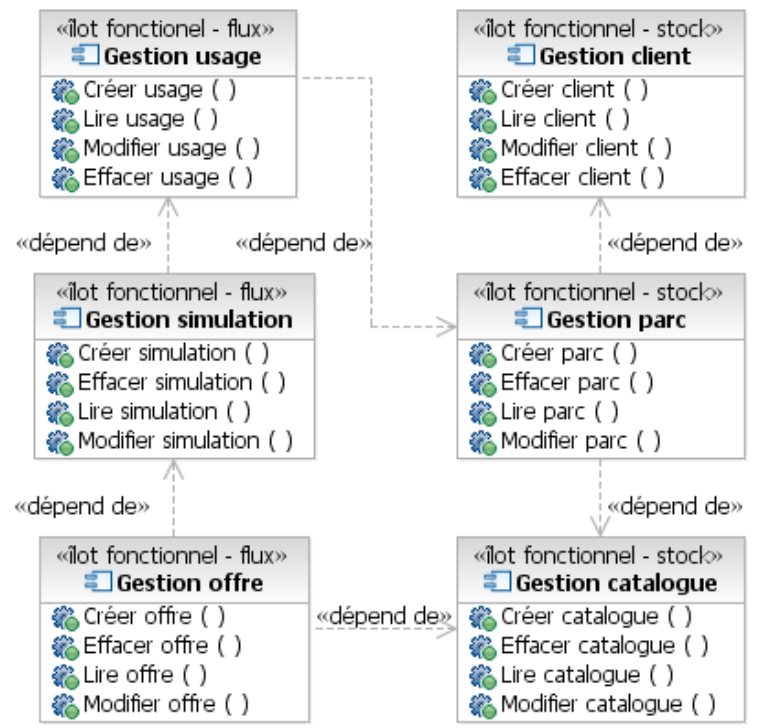

Figure 10. Extrait EA fonctionnelle cible liée aux offres

Les résultats des activités d'alignement statique et d'alignement dynamique du processus de complétion des offres avec l'EA fonctionnelle cible liée aux offres sont reportés, respectivement, dans les Tableaux 4 et 5 .

\begin{tabular}{|l|l|}
\hline \multicolumn{1}{|c|}{ Tâche métier } & Service fonctionnel \\
\hline Consultation du client & Lire client \\
\hline Consultation du parc du client & Lire parc \\
\hline Consultation de l'usage du client & Lire usage \\
\hline Création d'une simulation & Créer simulation \\
\hline Consultation du catalogue des offres & Lire catalogue \\
\hline Création d'une offre au client & Créer offre \\
\hline
\end{tabular}

Tableau 4. Illustration de l'alignement des tâches métier liées aux offres avec l'EA fonctionnelle cible 


\begin{tabular}{|l|l|}
\hline \multicolumn{1}{|c|}{ Succession entre tâches métier } & Dépendance entre îlots fonctionnels \\
\hline Consultation du client vers & Gestion parc vers \\
Consultation du parc du client & Gestion client \\
\hline Consultation du parc du client vers & Gestion usage vers \\
Consultation de l'usage du client & Gestion parc \\
\hline $\begin{array}{l}\text { Consultation de l'usage du client vers } \\
\text { Création d'une simulation }\end{array}$ & Gestion simulation vers \\
\hline Création d'une simulation vers & Gestion usage \\
Consultation du catalogue des offres & Aucune \\
\hline $\begin{array}{l}\text { Consultation du catalogue des offres vers } \\
\text { Création d'une offre au client }\end{array}$ & Gestion offre vers \\
\hline
\end{tabular}

Tableau 5. Illustration de l'alignement des successions de tâches métier liées aux offres avec l'EA fonctionnelle cible

La mesure de cet alignement est d'après [3] telle que :

$$
\operatorname{MAMF}\left(P_{\text {ComplétionOffres }}\right)=\left(\frac{6-0}{6}\right) *\left(\frac{5-1}{5}\right)=\frac{4}{5}
$$

Le choix du concepteur de services d'entreprise est pour cette application de ne pas corriger le processus métier. L'alignement avec l'EA fonctionnelle cible implique une rupture dans la réalisation du processus métier par les services d'entreprise. Cette rupture est localisée entre la gestion d'une simulation et la gestion d'une offre. La conception automatisée génère alors deux services d'entreprise de création d'une simulation et de création d'une offre entourés dans la Figure 11.

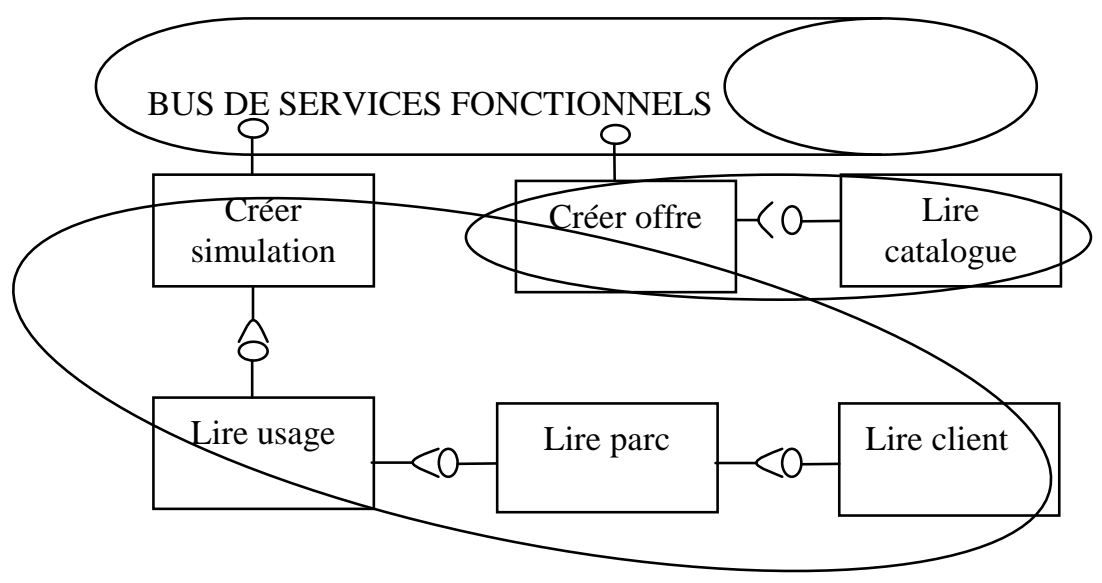

Figure 11. Services d'entreprise conçus pour les offres 


\section{Conclusion}

La démarche de conception de services d'entreprise proposée intègre l'EA fonctionnelle du SI cible. L'alignement du processus métier par rapport à l'EA fonctionnelle cible permet d'abord de faire un bilan de réutilisabilité potentielle d'éléments du SI. La vue fonctionnelle de tout élément du SI réutilisable doit être en effet conforme avec l'EA fonctionnelle cible. De ce fait, moins l'alignement est bon, plus le développement des services d'entreprise est coûteux.

De plus, un alignement parfait permet de réaliser un processus métier par un seul service d'entreprise. Moins cet alignement est bon, plus le nombre de services d'entreprises nécessaire augmente. Le coût du développement est donc plus important du fait de l'orchestration des services d'entreprise à mettre en œuvre.

Enfin, un bénéfice de l'adaptation de l'approche MDA proposée pour la conception des services d'entreprise est l'implémentation potentielle de l'algorithme de conception automatique des services d'entreprise avec des langages liés à l'ingénierie des modèles tels que ceux proposés dans KerMeta (Muller et al., 2005) ou ATL (Jouault et al., 2006).

\section{Bibliographie}

Booch G., Rumbaugh J., Jacobson I., «The Unified Modeling Language - User Guide », Addison - Wesley, 1999.

Burlton R.T., «Business Process Management - Profiting from Process», Sams Publishing, 2001.

Cauvet C., Guzelian G., «Business Process Modeling: a Service Oriented Approach », International Conference on System Sciences, Hawaï, 2008.

Code de l'urbanisme, «Plans locaux d'urbanisme», http://www.legifrance.gouv.fr/home.jsp, Article L123-1, 2008.

Gervais M.P., «Towards an MDA-Oriented Methodology », Proceedings of the $26^{\text {th }}$ Annual International Computer Software and Applications Conference (COMPSAC'02), 2002.

Grigoriu A., «SOA, BPM, EA, and Service Oriented Enterprise Architecture », BPTrends, www.bptrends.com, 2007.

Hammer M., Champy J., «Reengineering the Corporation: a Manifesto for Business Revolution », Harper - Collins, 1993.

Henderson J.C., Venkatraman N., «Strategic Alignment: Leveraging Information Technology for Transforming Organizations », IBM Systems Journal, vol. 32, No. 1, 1993, pp. 4-16.

Jouault F., Kurtev I., « On the Architectural Alignment of ATL and QVT », Proceedings of ACM Symposium on Applied Computing (SAC 06), Dijon, France, 2006. 
Kleppe A., Warmer J., Bast W., «MDA Explained - The Model driven Architecture: Practise and Promise », Object Technology Series, Addison-Wesley, 2003.

Kruchten P., «Architectural Blueprints - The "4+1" View Model of Software Architecture », IEEE Software, vol. 12, $\mathrm{n}^{\circ} 6,1995, \mathrm{p} .42-50$.

Longépé C., «Projet d'urbanisation du système d'information - Démarche pratique avec cas concret », Dunod/01 Informatique, 2001.

Miller J., Mukerji J., «MDA Guide Version 1.0.1 », http://www.omg.org/docs/omg/03-0601.pdf.

Muller P.A., Fleurey F., Jézéquel J.M., «Weaving executability into object-oriented metalanguages », Proceedings of MODELS/UML'2005, LNCS, Montego Bay, Jamaica, 2005.

OMG - MDA, « Model Driven Architecture (MDA)», http://www.omg.org/mda/.

Robinson R., «Understand Enterprise Service Bus scenarios and solutions in ServiceOriented Architecture », http://www-128.ibm.com/developerworks/webservices/library/ ws-esbscen/index.html.

Salinesi C., Thévenet L.H., «Enterprise Architecture, des problèmes pratiques à l'innovation », Ingénierie des systèmes d'information, vol. 13, Lavoisier, 2008, p. 75-105.

Schulte R.W., Natis Y.V., « Service Oriented Architectures - Part 1 », Gartner, 1996.

Shepperd M.J., Ince D., «Derivation and Validation of Software Metrics », Oxford University Press, 1993.

Simonin J., «Conception de l'architecture d'un système dirigée par un modèle d'urbanisme fonctionnel », thèse de doctorat mention informatique, Université de Rennes 1, 2009.

TMForum, « enhanced Telecom Operations Map », http://www.tmforum.org/ browse.aspx?catID=1648, 2009.

Wegmann A., «On the Systemic Enterprise Architecture Methodology (SEAM) », International Conference on Enterprise Information Systems (ICEIS), Angers, France, 2003.

Zachman J.A., «A Framework for Information Systems Architecture », IBM Systems Journal $26, \mathrm{n}^{\circ} 3,1987$, p. 276-292.

Zimmermann H., « OSI Reference Model - The ISO Model of Architecture for Open Systems Interconnection », IEEE Transactions on Communications COM-28, No. 4, 1980. 